\title{
COMPARISON OF A Nd: YAG LASER AND A XeCI LASER FOR THE ABLATION OF SOLIDS IN SPECTROCHEMICAL ANALYSIS
}

\author{
A. BRIAND, P. MAUCHIEN, F. CHARTIER* and J.M. MERMET* \\ SPEA/SPS/LSLA, Centre d'Etudes de Fontenay aux Roses, BP. 6, F-92265 Fontenay aux Roses cedex, \\ France \\ * Laboratoire des Sciences Analytiques, Université Claude Bernard Lyon I, \\ F-69622 Villeurbanne cedex, France
}

\begin{abstract}
As sample dissolution in elemental spectrochemical analysis is tedious and time consuming , there is therefore a need for direct solid analysis. Among the various possibilities, laser ablation seems to be attractive as it may be applied to conductive and non-conductive materials. Moreover a local analysis can be performed. Laser ablation can be used to remove material which is volatilized and excited (and ionized) in a secondary plasma source, such as an inductively coupled plasma (ICP) with either an optical or a mass detection. This method has never gained acceptance due to the selective volatilization of the elements of the target, resulting in interference effects. The reason for this is the use of high-power lasers, such as the ruby laser, which produce significant thermal effects.
\end{abstract}

Currently, there is a revival of laser ablation since the availability of reliable lasers with high repetition rate $(>10 \mathrm{~Hz})$ and an energy per pulse in the range of 0.1 to $0.5 \mathrm{~J}$, such as the Nd:YAG laser. More recent developments are based on the use of UV excimer lasers although almost no information is available on the capabilities of such lasers tor this purpose. We will indicate the influence of the laser characteristics, e.g. wavelength, beam protile, repetition rate and energy, on the ablation processes, in particular on the ablation efficiency for metais. Several metallic targets were selected so as to study the influence of the thermal properties of the metals. The excimer laser offers significant advantages over the Nd:YAG laser as it is less dependent on the properties of the metals. Analytical results and size of the particles which reach the plasma are discussed. Preliminary results concerning glass materials are also given. 